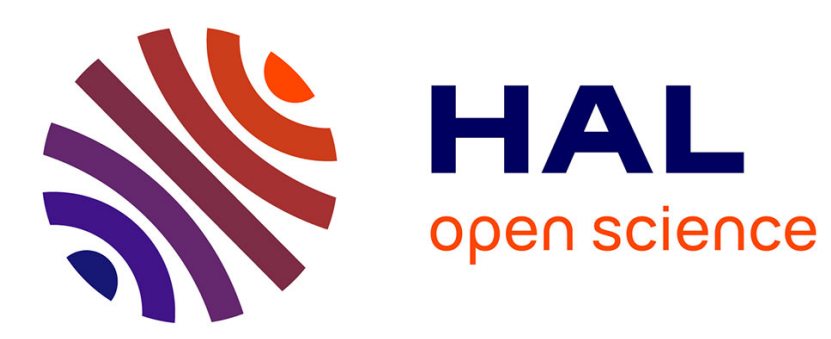

\title{
Le détachement d'objets indirects antéposés dans des énoncés à sujet inversé \\ Catherine Fuchs
}

\section{To cite this version:}

Catherine Fuchs. Le détachement d'objets indirects antéposés dans des énoncés à sujet inversé. Journal of French Language Studies, 2013, 23, pp.179 - 192. 10.1017/S095926951200004X . hal-01382589

\section{HAL Id: hal-01382589 \\ https://hal.science/hal-01382589}

Submitted on 17 Oct 2016

HAL is a multi-disciplinary open access archive for the deposit and dissemination of scientific research documents, whether they are published or not. The documents may come from teaching and research institutions in France or abroad, or from public or private research centers.
L'archive ouverte pluridisciplinaire HAL, est destinée au dépôt et à la diffusion de documents scientifiques de niveau recherche, publiés ou non, émanant des établissements d'enseignement et de recherche français ou étrangers, des laboratoires publics ou privés. 


\title{
Le détachement d'objets indirects antéposés

\author{
dans des énoncés à sujet inversé
}

\section{CATHERINE FUCHS}

ENS, PSL Research University, CNRS (LATTICE, UMR 8094), Paris 3

\begin{abstract}
L'étude porte sur les compléments en à et $d e$ placés à l'initiale d'un énoncé comportant une inversion du sujet nominal. Elle vise à comprendre pourquoi ces compléments sont parfois détachés à l'aide d'une virgule. L'observation de corpus textuels conduit à considérer que la présence ou l'absence de virgule signale deux constructions distinctes : dans la perspective théorique des approches macro-syntaxiques de l'énoncé en termes de 'noyau' / 'affixes', le complément initial suivi d'une virgule est analysé comme un préfixe détaché co-indexé au terme (vide) de départ du noyau, alors que dans la construction sans virgule le complément initial est intégré au noyau dont il occupe la première position. A cette différence syntaxique correspond une différence dans le mode de structuration informationnelle de l'énoncé (proposition catégorique ayant le complément pour thème et le noyau pour rhème et/ou focus $v s$. proposition thétique), qui elle-même induit deux types différents de stratégies discursives.
\end{abstract}




\section{INTRODUCTION}

La présente étude porte sur un type particulier de construction en français écrit. Il s'agit d'énoncés comportant une inversion du sujet nominal et, à l'initiale, un complément d'objet indirect (en à ou en de) détaché par une virgule (schéma ' $\mathrm{Ct}, \mathrm{VS}$ '). Exemples :

(1) A la déstabilisation de nos établissements, s'ajoutent désormais des modes d'action répréhensibles (...). (Conférence des Présidents d'Université, Le Monde, 23.04.2009, p. VII)

(2) De l'impasse où se trouvait l'entrée de l'hôtel, montait un chant d'accordéon. (F. Carco, Jésus-la-Caille, 1932 [1914], Albin-Michel, p. 24 ; cit. (E. Bordas, 2009 : 394)

L'inversion du sujet nominal en français - et tout particulièrement l'inversion dite 'locative' — a donné lieu à de nombreux travaux : voir par exemple Borillo (1990), Lahousse (2003), Marandin (2003), Fuchs (2006). Mais la question spécifique de la présence et du rôle de la virgule dans ce type d'énoncés ne semble guère avoir retenu l'attention des linguistes. Les grammaires normatives, quant à elles, considèrent que le tour 'correct' est le tour sans virgule. 
La question à laquelle nous nous attacherons est la suivante : le tour 'Ct, VS' (avec virgule) est-il différent du tour 'CtVS' (sans virgule)? En d'autres termes, la virgule est-elle syntaxiquement et/ou sémantiquement signifiante, ou bien le tour 'Ct, VS' (avec virgule) n'est-il qu'une variante du tour 'CtVS' (sans virgule) ? Pour tenter de répondre à cette question, notre étude se fondera sur l'observation d'un corpus écrit (principalement constitué d'articles de journaux, d'articles et d'ouvrages scientifiques, ainsi que d'exemples de la Toile et de quelques textes littéraires). Du fait qu'en français les verbes gouvernant un complément en $\grave{a}$ sont plus nombreux que ceux qui régissent un complément en de, le corpus contient $86 \%$ d'exemples avec un complément en $\grave{a}$ et $34 \%$ d'exemples avec un complément en de. Dans ce corpus, le tour sans virgule est majoritaire : il représente un peu plus de $64 \%$ des exemples, contre un peu plus de $35 \%$ pour le tour avec $\operatorname{virgule(s).}$

Seront exclus de l'étude les cas où le complément initial est un constituant complexe comportant un (ou des) ajout(s) (sous forme de relative, d'apposition, ou de juxtaposition) et où plusieurs virgules entourent ce (ou ces) ajout(s) :

(3) A cette politique restrictive, que les Etats candidats à l'élargissement ont dî intégrer au titre de l'acquis communautaire, fera certainement 
écho la politique restrictive des régions extra communautaires. (Corpus Chambers - Le Baron, 2006, LA-AD-NB-XX-XX-02)

(4) A la greffe, passée en quelques années du statut de technique de pointe transgressive à celui d'une technique de soin classique, s'ajoute une réflexion poussée qui a conduit la France à créer un statut de corps qui appartient à la collectivité et pas à l'individu. (Le Monde2, 31.05.2008, p. 66)

(5) D’un nuage gris, uniforme, diffus, dispersé aux quatre coins de l'univers, s'est différencié un système formé d'étoiles colorées. (Cl. Allègre, Introduction à une histoire naturelle, 1992, Fayard, p.80)

Dans des cas de ce genre, il est difficile en effet de savoir si la virgule entre le complément et la suite VS marque le bornage d'un constituant globalement détaché, ou bien si elle ferme des ajouts internes à ce constituant (et précédemment ouverts par une autre virgule) — auquel cas, la suppression des ajouts permettrait probablement de retrouver la structure sans virgule 'CtVS' :

(3') A cette politique restrictive fera certainement écho la politique restrictive des régions extra communautaires.

(4') A la greffe s'ajoute une réflexion poussée qui (...). 
(5') D’un nuage gris s'est différencié un système formé d'étoiles colorées.

C'est donc exclusivement aux cas où une seule virgule est présente que nous nous attacherons. Après avoir défalqué du corpus les exemples contenant plusieurs virgules, on obtient les proportions suivantes : 70\% d'exemples sans virgule et $30 \%$ d'exemples avec une virgule.

\section{LES CONSTITUANTS DE L'ÉNONCÉ EN PRÉSENCE DE LA VIRGULE}

Nous examinerons successivement les caractéristiques du complément initial, puis celles du sujet inversé. Les observations qui suivent permettent de dégager, non pas des règles absolues, mais des régularités (c'est-à-dire des tendances), qui signalent des affinités entre la présence de la virgule et certaines caractéristiques des constituants de l'énoncé.

\subsection{Le complément initial}

A première vue, on pourrait être tenté de corréler présence de la virgule et longueur du complément initial, comme semblent y inviter les exemples suivants : 
$(6)=((2)$ supra) De l'impasse où se trouvait l'entrée de l'hôtel, montait un chant d'accordéon. (F. Carco, Jésus-la-Caille, 1932 [1914], AlbinMichel, p. 24 ; cit. E. Bordas, 2009 : 394)

(7) A la faculté de retrait reconnue aux Etats dans le protocole $n^{\circ} 6$ (article 5), a été ajoutée la possibilité de modifier la déclaration. (Corpus Chambers - Le Baron, 2006, LA-AD-EC-XX-XX-03)

(8) A l'incapacité des lettrés et des clercs à reconnaître et à penser l'apparition d'un nouvel idiome dans les colonies, s'ajoute à la fin du $19^{e ̀ ~}$ s. européen la conceptualisation que la linguistique historique propose de ces langues. (D. Véronique, 2007. Des racines du langage : la linguistique naturaliste de Derek Bickerton, HEL, XXIX -2 : 173-174)

La virgule suit ici un constituant initial relativement long et complexe, qui ne se réduit pas à la seule tête nominale précédée de son déterminant (et éventuellement suivie d'un simple complément de nom). On pourrait penser que, dans l'hypothèse d'une telle réduction, la raison d'être de la virgule disparaîtrait :

(6') De l'impasse montait un chant d'accordéon.

(7') A la faculté de retrait a été ajoutée la possibilité de modifier la déclaration. 
(8') A l'incapacité des lettrés et des clercs s'ajoute à la fin du $19^{\grave{e}}$ s. européen la conceptualisation que (...).

Toutefois, outre le fait que les notions de longueur et de complexité sont (comme on le sait) difficiles à définir et délicates à manier, ce raisonnement se heurte à l'existence de contre-exemples. On trouve en effet des cas (assez rares, il est vrai) où un complément court est suivi d'une virgule :

(9) De Sagan, demeure, à jamais, un nombre non négligeable de textes très réussis. (Le Monde des livres, 20.06.2008, p. 2)

(10) Des bars de nuit, descendaient des fêtards et des femmes qui cherchaient une auto. (F. Carco, Jésus-la-Caille, 1932 [1914], AlbinMichel, p. 214 ; cit. E. Bordas, 2009 : 388)

Et surtout on rencontre, à l'inverse, nombre de cas où un complément relativement long n'est pas suivi d'une virgule :

(11) De ce nuage d'atomes projetés violemment dans toutes les directions de l'univers naîtront les galaxies et les étoiles. (Cl. Allègre, Introduction à une histoire naturelle, Fayard, 1992, p. 79)

(12) Aux chasseurs qui ont fait disparaître dès le XVIII $I^{e ̀}$ siècle les abondants troupeaux de baleines franches - bien trop lentes pour 
échapper aux harpons - ont succédé les mineurs de charbon puis, depuis une vingtaine d'années, les scientifiques et les touristes. (Le Monde, 06.06.2008, p. 23)

(Remarquons au passage que dans ce dernier exemple, le tiret n'équivaut pas à la virgule). ${ }^{1}$

Si la complexité interne et la longueur du complément (notions au demeurant tout à fait relatives) semblent donc pouvoir favoriser jusqu'à un certain point le recours à la virgule, on voit qu'elles ne le conditionnent pas pour autant.

Tournons-nous à présent vers le mode de détermination du complément. A l'exception d'un seul exemple, ${ }^{2}$ tous les énoncés du corpus (avec ou sans virgule) comportent un complément initial défini (le $\mathrm{N}, \mathrm{N}$ propre, ce $\mathrm{N}$, cela, en ou y). Les régularités que l'on observe concernent les compléments anaphoriques : on constate que plus le complément initial correspond à une anaphore stricte d'un élément du contexte antérieur, moins la virgule est attestée. Comme on pouvait s'y attendre, il n'y a jamais de démarcation graphique après les clitiques en et $y$ :

\footnotetext{
${ }^{1}$ En témoigne, a contrario, l'exemple suivant, qui cumule ces deux signes : A la fameuse question "qui a tué qui ? - celle des polars -, se substitue le très troublant "qui a engendré qui ? »-doute existentiel dont la réponse ne pousse pas toujours aux branches des arbres généalogiques, loin de là. (Le Monde des Livres, 15.05.2009, p.1).

${ }_{2}$ A des crimes contre l'humanité doit répondre une justice rendue au nom de la communauté internationale tout entière. (Corpus Chambers - Le Baron, 2006, LA-BF-ED$\mathrm{XX}-02-02)$.
} 
(13) Tout cela n'est pas faux. En atteste le riche volume collectif qui paraît sous la direction de Vincent Azoulay et Patrick Boucheron. (Le Monde des livres, 02.05.2009, p. 1)

(14) Tarif industriel 1. Tarif de gaz appliqué pour des besoins commerciaux, artisanaux ou industriels dont la puissance totale utile n'excède pas $150 \mathrm{~kW}$. Il se compose d'une prime fixe mensuelle par tranche entière et par tranche commencée de $5 \mathrm{~kW}$ de puissance installée, d'un prix de consommation en $€$ par $m^{3}$ de gaz. S'y ajoute un tarif pour la location du compteur qui inclut les frais de lecture annuelle du compteur et la facturation. (Google)

Il ne s'en trouve pas non plus, dans le corpus étudié, devant une anaphore en $\grave{a} /$ de cela :

(15) Dans la langue, le signifiant et le signifié donnent naissance au signe. De cela découle un autre signifiant prenant forme dans le mythe, et le signifié devient une "forme" associée à un nouveau "concept". (Google)

(16) Au fil des saisons est apparu un désaccord fondamental au sujet de l'âge minimal pour le recrutement des enfants et leur participation aux 
hostilités au sein des forces armées. A cela sont venues s'ajouter des questions importantes, à savoir les écoles militaires - et plus largement la formation militaire -, ainsi que le recrutement des enfants et leur participation aux hostilités au sein des groupes armés. (Corpus Chambers - Le Baron, 2006, LA-AD-JM-XX-XX-02)

C'est dans le cas des anaphores infidèles en ce $N$, que l'on observe une différence, selon qu'il y a ou non présence de la virgule; cette différence concerne le mode de reprise du contexte antérieur. Comparons les deux exemples suivants :

(17) «Depuis le début de l'année, les crédits automobiles ont chuté de 10\% », affirme Xavier Mosquet, directeur du Boston Consulting Group à Detroit (Michigan). A cette crise financière s'est ajoutée l'explosion du prix du gallon d'essence. De trois dollars le gallon (3,7 litres) en janvier, il est passé à près de quatre dollars en mai. (Le Monde, 29.05.2008, p. 15)

(18) Pour remédier à ce problème, les éditeurs ont défini une nouvelle clause stipulant que le pourcentage sera désormais calculé sur les rémunérations brutes du producteur, plus aisément identifiables. De même, afin de "valoriser » les sujets, les éditeurs entendent indexer l'àvaloir sur le budget du film. A cette première pierre jetée dans le jardin 
des agents, s'ajoute celle des droits d'adaptation. (Le Monde des livres, 15.05.2009, p. 5)

A première lecture, les deux exemples semblent très proches puisqu'ils contiennent un complément initial formellement comparable ( $a$ cette $N$ ) et le même lexème verbal (s'ajouter). Toutefois, il apparaît que le mode de 'recatégorisation' opéré par cette anaphore dite 'démonstrative"3 n'est pas exactement le même. En (17), cette crise financière recatégorise l'état de choses décrit dans l'énoncé précédent (la chute de 10\% des crédits automobiles) à l'aide d'une équivalence présentée comme 'objective' : la chute des crédits est l'indice d'une crise financière. En (18), cette première pierre jetée dans le jardin des agents recatégorise le contenu des énoncés précédents par le biais d'une requalification plus amplement développée et davantage 'subjective' : le pourcentage des droits et l'indexation de l'àvaloir, décidés par les éditeurs, sont jugés par le journaliste constituer une première pierre jetée dans le jardin des agents.

Le même processus se retrouve dans l'exemple suivant, où l'état de choses décrit dans le contexte antérieur (en l'occurrence, l'attitude du gouvernement israélien) est repris par le journaliste à l'aide d'une anaphore infidèle qui requalifie subjectivement cet état de choses, en l'assimilant à une forme d'autisme politique :

${ }^{3}$ Sur la notion d'anaphore démonstrative, voir notamment Corblin (1987) et Kleiber (1994). 
(19) L'exigence d'un état répond à un droit du peuple palestinien. S'il est logique de négocier les conditions de l'avènement de cet Etat, il est indécent d'en nier la légitimité. C'est pourtant ce que fait le gouvernement israélien, qui prétend convaincre le monde qu'il s'agit d'un problème secondaire, au regard du danger que représente l'industrie nucléaire iranienne. A cette forme d'autisme politique, doit répondre une mobilisation des opinions - pour peser dans le sens des réalités et sauver la chance de conclure une vraie paix. (Le Monde, 05.06.2009, p. 21)

Pour résumer concernant les cas d'un complément initial anaphorique : toujours absente après un complément anaphorique strict, la virgule suit préférentiellement un complément anaphorique de type requalification 'subjective' du référent (plutôt qu'un complément anaphorique de type redénomination 'objective' d'un référent précédemment introduit).

\subsection{Le sujet inversé}

S'agissant des caractéristiques du sujet inversé dans le tour avec virgule, on observe deux types de régularités. Tout d'abord, la présence de la virgule après $\mathrm{Ct}$ s'avère favoriser l'expression d'un contraste entre le référent de ce 
complément et celui du sujet, lorsque ceux-ci participent tous deux d'un même domaine. ${ }^{4}$ Si l'on reprend les exemples (17) et (18) ci-dessus, on voit qu'en (17), le sujet introduit un référent (l'explosion $d u$ prix $d u$ gallon d'essence) distinct de celui auquel correspond le complément (cette crise financière), alors qu'en (18), au contraire, le référent du sujet et celui du complément sont des occurrences d'une même classe (une première pierre ... et celle des droits d'adaptation, c'est-à-dire une autre pierre ...).

Ce même fonctionnement se retrouve dans l'exemple suivant, où sont mises en regard une extension conceptuelle $(\mathrm{Ct})$ et une extension matérielle (S) :

(20) A la lumière de ces brèves indications, on ne peut que souligner l'élargissement sensible $d u$ concept de discrimination en droit communautaire qui vient ainsi utilement compléter la protection issue de l'article 14 de la CEDH. Cette extension doit être saluée en ce qu'elle vise à répondre à des atteintes concrètes, parfois peut-être même inconscientes pour ce qui concerne la discrimination " indirecte 》, au principe d'égalité, qui contribuaient à remettre en question l'efficacité de la lutte contre les discriminations. A cette extension conceptuelle, correspond une extension matérielle $d u$ principe de nondiscrimination. (Corpus Chambers - Le Baron, 2006, LA-DF-EB-XX02-02)

${ }^{4}$ On retrouve ici la notion de 'contraste' fondée tout à la fois sur la similarité et la dissimilarité dans le parallélisme, telle que la présente Umbach (2004) à propos du focus. 
et aussi dans celui-ci, où sont contrastés le désespoir écrasant $(\mathrm{Ct})$ et le remords plus écrasant encore (S) :

(21) Au désespoir écrasant de l'avoir perdu, se mêlerait le remords plus écrasant encore d'avoir causé sa perte. (H. Troyat, Le vivier, II, ch. 5, p. 98 ; cit. Le Bidois, 1952 : 157)

Par ailleurs, la présence de la virgule après $\mathrm{Ct}$ s'avère favoriser l'élaboration progressive du référent de $\mathrm{S}$, via diverses qualifications du nom-tête, du type un (autre) $N:$ (=à savoir) :

(22) Au débat génétique sur les créoles, s'ajoute une autre thématique, [= à savoir] celle de leur jeunesse et de leur simplicité, manifestées par une absence de morphologie. (D. Véronique, art. cit. : 173-174)

(23) De ces lectures faites trop tôt, et d'un vagabondage irrévérencieux dans les massifs de pages, me sont longtemps restées des images plus que des idées : [= à savoir] dans les Paroles d'un croyant, les rois agrippés à leurs couronnes, comme si [...] ; dans les Mémoires d'outretombe, la tourelle solitaire de Combourg et la canne du père résonnant 
dans les couloirs glacés [...]. (M. Ozouf, Composition française, 2009, Gallimard, p. 94)

(24) (= (1) supra) A la déstabilisation de nos établissements, s'ajoutent désormais des modes d'action qui sont aux antipodes des valeurs universitaires de dialogue et de respect: [= à savoir] agressions physiques et verbales des responsables et des personnels universitaires, annonces fracassantes de délivrance complaisante de diplômes, entraves au droit d'étudier librement, harcèlement systématique des instances délibératives et décisionnelles dans les établissements, détérioration des locaux. Nous ne pouvons pas accepter que s'installe une telle violence dans des lieux qui ne vivent que par la liberté et la tolérance intellectuelles. (Conférence des Présidents d'Universités, Le Monde, 23.04.09, p. VII)

Il arrive même que cette élaboration progressive du référent de S se poursuive dans les énoncés ultérieurs :

(25) (= 9 supra) De Sagan, demeure, à jamais, un nombre non négligeable de textes très réussis. [= à savoir] En premier lieu, Bonjour tristesse. Mais, si l'on n'a encore rien lu d'elle, on peut commencer par ses deux magnifiques exercices d'admiration - une qualité rare, que possédait Sagan —, sorte d'autobiographie en fragments : Avec mon meilleur souvenir (Gallimard, 1984), ... et toute ma sympathie (Julliard, 1993). Ce sont deux petits bijoux d'élégance d'esprit, de 
délicatesse, de passion. Avec, toujours, son style rapide, sans afféteries. (Le Monde des livres, 20.06.2008, p. 2)

$\mathrm{Au}$ contraire, l'absence de virgule après Ct s'observe dans les cas où le référent de $\mathrm{S}$ est donné d'emblée comme autonome (groupe nominal en un/le $N$ ), qui n'appellera pas de développement dans les énoncés ultérieurs :

(26) Aux produits précédents s'ajoutent en abondance les silicates. (Cl. Allègre, Introduction à une histoire naturelle, 1992, Fayard, p. 120) (Contrairement aux exemples (18) et (22) supra, qui contiennent le même verbe s'ajouter, on voit qu'en (26), le sujet est donné comme référentiellement autonome et n'est pas mis en contraste avec le complément, et que ce dernier n'est pas une anaphore infidèle 'subjective'.)

(27) Sans attendre la fin du rattrapage, l'heure est donc venue d'agir afin d'occuper une place de leader dans la course au numérique. De ce classement dépendra la part de la croissance économique dont la France pourra bénéficier. (Le Monde, 30.07.2008, p. 2)

En résumé : la présence de la virgule après Ct favorise l'expression d'un contraste entre le référent de ce complément et celui du sujet, et/ou 
l'élaboration progressive du référent de S. Autant d'indices en faveur d'un fonctionnement du sujet comme rhématisé ou mis en focus, lorsque le complément initial est suivi d'une virgule.

\section{LA STRUCTURATION DE L'ÉNONCÉ}

Des observations qui précèdent il découle que la présence d'une virgule après le complément initial révèle certaines spécificités de ce complément et, plus largement, donne des indications sur le mode de structuration de l'énoncé.

Pour interpréter ces résultats, nous nous situerons dans le cadre d'une approche de l'énoncé en termes de 'noyau' et de 'périphériques' (ou 'affixes'), telle qu'illustrée notamment par Le Goffic (2008). L'idée de base est de décrire l'énoncé comme constitué d'un noyau prédicatif qui peut être précédé de constituants pré-noyau ('préfixes'), et/ou être suivi de constituants post-noyau ('suffixes'), et/ou comporter en son sein des constituants extra-prédicatifs en incises ('infixes'). Schématiquement: Enoncé $=($ Préfixes $)+[$ Noyau $+($ Infixes $)]+($ Suffixes $)$.

Dans ce cadre théorique, il convient de distinguer les deux notions de 'place' et de 'position' (Fuchs et Le Goffic, 2007). En effet, une 'position' (comme, par exemple, celle de terme de départ du noyau, ou celle de préfixe antéposé au noyau) est une place fonctionnellement 'étiquetée', 
c'est-à-dire une place dotée d'un fonctionnement syntaxique spécifique au sein d'une construction donnée: la perspective est topologique. En revanche, une 'place' (non étiquetée) correspond simplement à un placement de surface (comme par exemple le fait, pour un constituant, d'être placé à l'initiale de l'énoncé) : la perspective est purement linéaire. Le noyau d'un énoncé est constitué au minimum d'un terme de départ (occupant la première position) et du verbe (en seconde position) - ces deux constituants pouvant être suivis d'un ou plusieurs complément(s) et/ou d'un ou plusieurs adjoints intra-prédicatif(s). Schématiquement : Noyau $=$ Terme de départ + Verbe $(+\mathrm{Ct}(\mathrm{s})(+\operatorname{adjoint}(\mathrm{s})$ intra-prédicatif(s)). Lorsque le terme de départ du noyau n'est pas le sujet (S) mais un autre constituant intra-prédicatif (complément- ${ }^{5}$ ou adjoint ${ }^{6}$ ), comme c'est le cas des énoncés ici étudiés, alors le sujet nominal ${ }^{7}$ est inversé.

Les régularités observées sur corpus conduisent à considérer que la place à l'initiale de l'énoncé occupée par le complément n'a pas le même statut, en termes de position fonctionnelle, selon que ce complément est, ou non, suivi d'une virgule. Suivi de la virgule, il fonctionne comme un constituant topicalisé détaché par l'énonciateur (d'où l'affinité avec une

\footnotetext{
${ }^{5}$ Attribut du sujet : (Grande fut ma surprise) ou objet indirect (De cette lettre dépend mon avenir ; A cette époque heureuse succèdera une ère de misère). On notera au passage que le français moderne ne permet plus de prendre comme terme de départ un objet direct ( ${ }^{*} L a$ chanson chantait mon père) - cette position n'étant conservée qu'en subordonnée ( $\mathrm{La}$ chanson que chantait mon père).

${ }^{6}$ Compléments de temps (A 20 heures arrivèrent les officiels), compléments de lieu (Dans cette maison naquit Victor Hugo) ou compléments de manière (Ainsi finit l'histoire).

${ }^{7}$ Cette inversion ne concerne que le sujet nominal, c'est pourquoi le sujet clitique n'est pas considéré ici.
} 
anaphore infidèle de type requalification 'subjective') ; et c'est à propos de ce topique que le reste de l'énoncé est prédiqué comme rhème (d'où l'affinité avec l'élaboration progressive du sujet) et/ou comme focus (d'où l'affinité avec un contraste entre complément et sujet). Plus précisément, nous poserons que la place initiale du complément détaché par la virgule correspond à une position de périphérique extérieur au noyau - ce qui le différencie du complément intégré, dont la place initiale coïncide avec la position de terme de départ du noyau; mais, contrairement au cas des périphériques appelés 'cadratifs' par Charolles (2003), le constituant détaché Ct reste lié au noyau, par le biais d'un mécanisme de co-indexation.

Dans cette perspective, les deux structures de surface 'Ct, VS' et 'CtVS' sont à considérer comme deux 'constructions' (au sens de Goldberg, 2006) distinctes. Dans le cas de la construction sans virgule, 'CtVS' constitue globalement un noyau ; dans le cas de la construction avec virgule, la première position au sein du noyau est vide ( ) et le Ct détaché est co-indexé avec cette position (qui, dans bon nombre de cas, pourrait être réalisée sous la forme du il impersonnel ${ }^{8}$ ); d'où le schéma 'Ct, ( )VS'. On remarquera au passage que notre analyse rejoint à certains égards celle proposée par Sabio (2006) et par Abeillé, Godard \& Sabio (2008) à propos d'un type particulier de compléments d'objets directs disloqués à gauche, dits 'antéposés sous forme de préfixes macro-syntaxiques', comme : Le

\footnotetext{
8 Sur le 'test' de l'insertion possible du il impersonnel, voir Cornish (2005 : section 5).
} 
chocolat, j'aime. Par différence avec le type Huit ans, il a ('compléments antéposés sous forme de noyau'), les compléments du premier type sont décrits par ces auteurs comme des 'périphériques liés' fonctionnant comme thème d'une proposition catégorique. Le liage est dit opérer entre le complément nominal antéposé et un 'complément pronominal nul, présent comme argument, mais sans réalisation phonique'.

Dans notre perspective, le noyau à sujet inversé correspond, dans la construction sans virgule, à un jugement de type 'thétique', selon la terminologie d'Anton Marty, reprise par Kuroda (1973). 'CtVS' fonctionne comme un noyau globalement thétique ayant pour terme de départ le complément essentiel, à l'instar des inversions locatives où le terme de départ est un complément accessoire intra-prédicatif : voir Cornish (2001). D'où la représentation suivante, où le symbole $<>$ encadre le noyau :

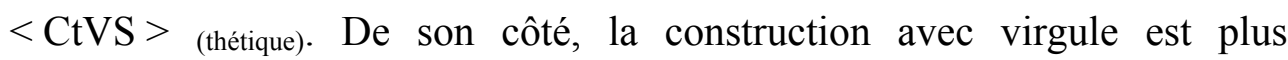
complexe et correspond, dans son ensemble, à un jugement de type 'catégorique'. En effet, le complément détaché fonctionne comme un préfixe thématique dont le noyau thétique $<(\mathrm{)VS}>$ à premier terme vide constitue alors le rhème. D'où la représentation : $[\mathrm{Ct},]_{\text {(thème) }}[<(\mathrm{VS}>$ (thétique)] (rhème). On a donc affaire ici à une construction informationnelle particulière, à savoir une construction thétique, globalement rhématique, enchâssée dans une construction catégorique dont le complément constitue 
le thème. Ce type d'enchâssement a déjà été proposé, à propos de divers types d'énoncés, par certains auteurs ${ }^{9}$.

Ce fonctionnement spécifique du complément détaché est sans doute à relier au fait qu'il admet difficilement d'être précédé d'un autre périphérique topicalisé (comme, par exemple, un complément 'cadratif' spatio-temporel) : de fait, aucun exemple du type ??? Après cette victoire, à ses premiers succès, s'est ajoutée la reconnaissance de ses pairs ne figure dans le corpus étudié. Au contraire, on trouve des exemples où 'CtVS' (sans virgule après $\mathrm{Ct}$ ) est précédé d'un tel périphérique :

(28) Dans la France de 1940, au chagrin de la défaite s'ajoutait pour moi l'angoisse de constater, ça et là, une certaine complicité avec elle [...]. (G. Tillion, Fragments de vie, 2009, Le Seuil, p. 128)

La construction d'un énoncé en 'CtVS' se fait en une seule étape, ce qui permet de construire une vue globale 'objective' de la situation décrite. A l'inverse, la construction d'un énoncé en 'Ct, VS' se fait en deux étapes. ${ }^{10}$ Un thème détaché $(\mathrm{Ct})$ est d'abord posé, qui entraîne ensuite l'instauration d'un rhème, constitué par le noyau à première position vide ; d'où une sorte de mise en scène 'subjective' de la situation. On pourrait

${ }^{9}$ Ainsi, par exemple, Tasmowski \& Willems (1987: 186) opposent-elles ' $I l$ VS', décrit comme "un énoncé thétique simple», et 'Loc (il) VS' qui est dite "emboîter une construction thétique ((il)VS) dans un énoncé catégorique (Loc (il) VS)».

${ }^{10}$ Anton Marty parlait déjà de 'jugement double' à propos du jugement catégorique, par opposition au 'jugement simple' que constitue le thétique. 
transposer, en l'adaptant à 'Ct, VS', la formulation proposée par Tasmowski et Willems (1987: 184) à propos de 'Loc (il) VS': il s'agirait d'une 'présentation en deux mouvements', comparable au 'réglage progressif d'une longue-vue'.

\section{LE TISSAGE DE LA TRAME DISCURSIVE}

Comme nous allons le voir maintenant, ces deux modes différents de structuration de l'énoncé se reflètent dans le tissage de la trame discursive. Selon que la virgule est présente ou non, on observe en effet des différences au plan de la conduite du discours. Deux stratégies distinctes se révèlent être à l'œuvre.

Dans le cas de 'CtVS', l'énoncé assure la transition entre le contexte antérieur et le contexte ultérieur ; il opère, au plan référentiel, ce que nous avons appelé un double 'chaînage discursif' : un 'chaînage arrière' à l'aide de Ct et un 'chaînage avant' à l'aide de S (Fuchs, sous presse). Cela a pour effet d'assurer une progression quasi-linéaire du discours. L'exemple qui suit, consacré à la présentation d'un calendrier astronomique et olympique vieux de plus de deux mille ans (titre de l'article), illustre bien cette stratégie :

(29) L'appareil, contenu dans un boîtier en bois haut de $33 \mathrm{~cm}$, large de $18 \mathrm{~cm}$ et épais de $10 \mathrm{~cm}$, était vraisemblablement actionné par une 
manivelle. A l'avant figuraient deux cadrans représentant le calendrier égyptien et le Zodiaque grec. A l'arrière étaient placés deux autres cadrans, correspondant au cycle métonique (période de dix-neuf ans, soit 235 lunaisons, au terme de laquelle les phases de la Lune reviennent aux mêmes jours de l'année) et au cycle de Saros (permettant de calculer le retour des ellipses lunaires et solaires).

Au cadran métonique est associé un petit cadran subsidiaire, que les chercheurs croyaient lié au cycle callipique (période de $4 \times 19$ ans). Or de nouvelles analyses ont montré qu'il indiquait, en réalité, la séquence des olympiades grecques et leur correspondance avec les années civiles. (Le Monde, 01.08.2008, p. 7)

Dans le premier paragraphe sont introduits successivement deux cadrans (situés à l'avant de l'appareil) et deux autres cadrans (placés à l'arrière) correspondant, l'un au cycle métonique, et l'autre au cycle de Saros. Le second paragraphe commence par un énoncé en ' $\mathrm{CtVS}$ ', où $\mathrm{Ct}$ reprend 'le cadran métonique' et $\mathrm{S}$ permet d'introduire un nouveau référent ('un petit cadran subsidiaire') qui dès lors devient l'objet saillant dans la suite du discours (il est repris par que dans le même énoncé, puis par il dans l'énoncé suivant). Il y a donc bien chaînage discursif: l'énoncé 'CtVS' constitue l'étape intermédiaire qui assure la transition entre deux objets de 
discours en faisant passer du 'cadran métonique' au 'petit cadran subsidiaire'.

Il n'en va pas de même dans le cas de 'Ct, VS', où s'opère une autre stratégie, que nous avons qualifiée de '(ré)amorçage discursif' (Fuchs, sous presse). Au lieu d'une progression linéaire du discours, c'est plutôt à un arrêt permettant une sorte de déploiement interne que l'on assiste. Considérons l'exemple suivant :

(30) Au-delà des nationalismes, des chauvinismes plutôt, s'affrontent deux théories sur la nature même du sport. A l'idée d'une pratique hygiéniste et formatrice, s'oppose celle d'un vecteur d'émotions et de passions. Pour les uns prévaut l'équité. Pour les autres l'excellence. Avec cette sempiternelle question : peut-on avoir à la fois la morale et les victoires ? (Le Monde, 03-04.08.2008, p. 2)

Dans le premier énoncé sont introduits deux objets ('deux théories'). Au lieu de faire passer de ces objets à d'autres, l'énoncé en 'Ct, VS' marque au contraire un temps d'arrêt sur ces objets, en les détaillant et en les contrastant l'un à l'autre : Ct décrit la première théorie (l'idée d'une pratique hygiéniste et formatrice), S la seconde (celle d'un vecteur d'émotions et de passions). Et la suite du discours, loin d'enchaîner sur l'objet décrit par S, développe au contraire l'opposition entre ces deux objets : pour les uns, l'équité vs. pour les autres, l'excellence; la morale vs. 
les victoires. Ce n'est donc pas à une progression du discours que l'on assiste, mais bien plutôt à la mise en scène d'une situation décrite et qualifiée 'subjectivement' de façon réitérée sous des angles divers; on notera en particulier le recours aux couples de quasi-synonymes (équité/morale, excellence/victoires) à travers lesquelles le journaliste tente progressivement de spécifier les propriétés des deux théories en question.

\section{CONCLUSION}

Pour ténue qu'elle puisse apparaître au premier abord, la différence entre 'Ct, VS' et 'CtVS' n'en est pas moins réelle : toute une série de régularités atteste qu'il y a bien là à l'œuvre deux mécanismes distincts, producteurs d'effets différents.

L'étude a en effet permis d'établir que la place initiale du complément détaché par la virgule correspond à une position de périphérique extérieur au noyau co-indexé avec la première position (vide) du noyau - ce qui le différencie du complément sans virgule, dont la place initiale coïncide avec cette position. En conséquence, la structure de surface 'Ct, VS' constitue une construction sui generis, distincte de la construction canonique 'CtVS'. Dans 'Ct, VS', la première position au sein du noyau est vide ; le complément est posé à l'initiale comme un thème détaché, ce qui entraîne la rhématisation du noyau thétique. D'où une stratégie de 
'(ré)amorçage discursif', distincte de la stratégie de 'chaînage discursif' qui caractérise la construction sans virgule 'CtVS'.

Il va de soi que cette différence n'est sans doute pas consciente dans l'esprit des scripteurs, pas plus qu'elle ne l'est dans celui des lecteurs. Pour autant, il n'est pas exclu que les sujets soient sensibles au fait que la virgule après le complément permet d'autonomiser un objet thématique subjectivement investi, autour duquel peut s'instaurer, voire se prolonger, toute une mise en scène.

La question de l'apparition historique de ce type de construction à virgule reste à explorer, ainsi que celle des registres et types de discours qui le favorisent. Si l'on constate à l'heure actuelle un recours assez massif à 'Ct, VS' dans les articles de journaux (au point d'être tenté d'y voir une sorte de 'mode' ou de 'tic' d'écriture), il n'en reste pas moins que cette construction se rencontre également sous la plume d'écrivains, y compris et c'est là le point intéressant - chez ceux qui produisent concurremment, dans un même texte, des énoncés en 'CtVS'. Tel est le cas de Proust (les exemples ci-dessous sont repris de Le Bidois, 1952 : 158-159), chez qui l'on trouve aussi bien les constructions à complément détaché :

(31) Au regard que je venais de poser sur telle ou telle d'entre elles, s'appariait immédiatement le regard curieux, furtif, entreprenant, [...] $(\mathrm{XIV}, 12)$ 
(32) Aux différences qu'il y avait entre eux, étaient bien loin de correspondre sans doute les différences $[\ldots](\mathrm{V}, 246)$

que les constructions à complément lié :

(33) A son mépris pour Saint-Loup s'ajouta la même haine que s'il s'était obstiné à vouloir lui inculquer une maladie mortelle $(\mathrm{V}, 28)$

(34) Car aux troubles de la mémoire sont liées les intermittences du cour (VIII, 178)

La présence d'une virgule après le constituant initial de l'énoncé ne touche d'ailleurs pas seulement les compléments. On la retrouve aussi dans le cas des adjoints, notamment dans celui des inversions locatives (spatiales ou temporelles) ${ }^{11}$ : sur ce point (voir Fuchs, sous presse). En voici deux exemples :

(35) Sur la table du directeur de l'école, trônent une cinquantaine de lettres d'encouragement. (Le Monde, 30.05.2008, p. 28)

(36) Après l'ondée torrentielle de la nuit, brille un soleil ardent. (T. Raucat, L'honorable partie de campagne, 1984 [1927], Gallimard Folio, p. 1)

11 Cela n'a rien d'étonnant, si l'on admet, comme le fait par exemple Cornish (2005: section 2), qu'il existe une gradation des relations unissant le constituant initial (complément ou adjoint) et le verbe placé devant le sujet. 
S'agirait-il là d'une stratégie discursive en expansion, voire de l'indice d'une évolution de la langue ? Ce développement, à l'écrit, de la préfixation de constituants essentiels ou accessoires intra-prédicatifs serait-il influencé par certaines structures de la langue orale? Autant de questions qui mériteraient d'être approfondies.

Adresse de l'auteur pour correspondance:

Catherine Fuchs,

Lattice,

Ecole Normale Supérieure,

1 rue Maurice Arnoux

92120 Montrouge

Email: <catherine.fuchs@ens.fr>

\section{REFERENCES}

Abeillé, A., Godard, D. et Sabio, F. (2008). Deux constructions à SN antéposé en français. Dans: J. Durand, B. Habert et B. Laks (dir.), Congrès Mondial de Linguistique Française. Paris: Institut de Linguistique $\quad$ Française. $\quad$ CD-Rom : 2349-2364. (http://dx.doi.org/10.1051/cmlf08210). 
Bordas, E. (2009). Les inversions des invertis ?. Dans: A. FontvieilleCordani et S. Thonnerieux (dir.), L'ordre des mots à la lecture des textes. Lyon: Presses Universitaires de Lyon, pp. 387-402.

Borillo, A. (1990). A propos de la localisation spatiale. Langue française, 86: $75-84$.

Chambers, A. et Le Baron, F. (2006). Corpus d'articles de recherche en français (version électronique).

Charolles, M. (2003). De la topicalité des adverbiaux détachés en tête de phrase. Travaux de Linguistique, 47: 11-51.

Corblin, F. (1987). Indéfini, défini et démonstratif. Paris: Droz.

Corblin, F. (1995). Les formes de reprise dans le discours : anaphores et chaînes de référence. Rennes: Presses de l'université de Rennes.

Cornish, F. (2001). L'inversion 'locative' en français, italien et anglais. Cahiers de Grammaire, 26: 101-123.

Cornish, F. (2005). A cross-linguistic study of so-called 'locative inversion' : evidence for the Functional Discourse Grammar model. Dans: C. de Groot et K. Hengeveld (dir.), Morphosyntactic Expression in Functional Grammar. Berlin et New-York : Mouton de Gruyter, pp. 163202.

Fuchs, C. (2006). Locatif spatial initial et position du sujet nominal : pour une approche topologique de la construction de l'énoncé. Linguisticae Investigationes, XXIX-1: 61-74. 
Fuchs, C. (sous presse ; à paraître en 2012). A paradoxical case of locative inversion. Dans: L. Sarda, S. Carter-Thomas et B. Fagard (dir.), Markers of Discourse Organisation: word order parameters and the structuring role of adverbials. Louvain-la-Neuve: UCL Publishers, coll. Language and Corpora in Use.

Fuchs, C. et Le Goffic, P. (2007). Le français moderne : entre «V2» et « SVO » ?. Dans: O. Bertrand, S. Prévost, M. Charolles, J. François et C. Schnedecker (dir.), Discours, diachronie, stylistique du français. Berne: Peter Lang, pp. 17-35.

Goldberg, A. (2006). Constructions at Work: the nature of generalization in language. Oxford: Oxford University Press.

Kleiber, G. (1994). Anaphores et pronoms. Louvain-la-Neuve: Duculot.

Kuroda, S-Y. (1973). Le jugement catégorique et le jugement thétique : exemples tirés de la syntaxe japonaise. Langages, 30: 81-110.

Lahousse, K. (2003). La complexité de la notion de topique et l'inversion du sujet nominal. Travaux de Linguistique, 47: 111-136.

Le Bidois, R. (1952). L'inversion du sujet dans la prose contemporaine. Paris: d'Artrey.

Le Goffic, P. (2008). Phrase, séquence, période. Dans: D. Van Raemdonck (dir.), Modèles syntaxiques : la syntaxe à l'aube $d u X X I^{e}$ siècle. Bruxelles: Peter Lang, pp. 329-356. 
Marandin, J-M. (2003). Inversion du sujet et discours dans les langues romanes. Dans: D. Godard (dir.), Les langues romanes : problèmes de la phrase simple. Paris: CNRS éditions, pp. 345-392.

Sabio, F. (2006). L'antéposition des compléments en français contemporain : l'exemple des objets directs. Linguisticae Investigationes, XXIX-1: 173-182.

Tasmowski, L., et Willems, D. (1987). Les phrases à première position actancielle vide. Travaux de Linguistique, 14-15: 177-191.

Umbach, C. (2004). On the Notion of Contrast in Information Structure and Discourse. Journal of Semantics, 21-2: 1-21. 
[Version pre-print d'un article paru en 2013 dans :

Journal of French Language Studies 23 :2, pp. 179-192.] 